\title{
Virtual Laboratory of Robot Kinematics Based on Qt and Ogre3D
}

\author{
Fei Ma ${ }^{a}$, Zhiyuan Sun ${ }^{b}$ and Ruiqing $\mathrm{Jia}^{\mathrm{c}}$ \\ School of Mechanical Electronic \& Information Engineering, China University of Mining \& \\ Technology Beijing, China \\ amashengbo1990@126.com, b353642795@qq.com, cruiqingjia@yahoo.com
}

\begin{abstract}
Keywords: Virtual laboratory, Kinematics, Robotics, Virtual experiment.
\end{abstract}
\begin{abstract}
This paper describes the design of a virtual laboratory based on the Qt and Ogre3D to meet the experimental requirements in the Robot Kinematics. In this paper, a six-degree-of-freedom industrial robot kinematics virtual experiment is created by virtual laboratory platform based on the Qt and Ogre3D as a part of the virtual laboratory of robotics. Coordinate system arrangement and kinematics of the six-degree-of-freedom industrial robot are illustrated in the 3D environment intuitively and clearly. The experimental results show that the virtual experiment can make the teaching process more reasonable and show that the virtual laboratory will play an important role in the future of robotics teaching.
\end{abstract}

\section{Introduction}

Virtual Laboratories (hereinafter referred to as VLs) use computer technology to virtualise real experimental environment and experimental content, which can achieve same experimental results as actual experiment.

In the field of robots, there are a large number of universities at home and abroad engaged in virtual laboratory research. Carlos A. Jara and Francisco A. Candelas use EJS software and EjsRL library to create virtual robot applications from two levels of architecture. EJS provides 2D / 3D graphical interface, EjsRL provides a function for modeling, simulation and computer vision algorithms. The combination of the two forms the software architecture of VLs [1]. University of Hernandez, Spain use Easy Java Simulation to develop teaching of robot virtual laboratory in the research of the LABEL project [2]. In the VCIMLAB project that provides a 3D interactive virtual laboratory environment, Cyprus East Mediterranean University trains using industrial robots, CNC machine tools and automatic assembly of equipment in Computer integrated manufacturing(CIM) [3].

With the development of virtual laboratories, VLs are widely used in different areas [4], and have more and more relevant research literature. Due to the mechanical and electrical integration characteristics and multidisciplinary nature of robotics, robotics becomes the best example of using virtual and remote laboratory teaching. However, few VLs focus on rigid body motion and coordinate transformation of robot kinematics.

In this paper, a six-degree-of-freedom industrial robot kinematics virtual experiment is created by virtual laboratory platform based on the QT and Ogre3D as a part of the virtual laboratory of robotics. Coordinate system arrangement and kinematicsof the six-degree-of-freedom industrial robot are illustrated in the 3D environment intuitively and clearly.

\section{Virtual Laboratory Architecture}

\subsection{The Software Architecture of Virtual Laboratory of Kinematics}

The architecture of VLs platform based on Qt and Ogre3D using modular structure is shown in Figure 1. Qt is responsible for the display of interactive parts and hardware interaction part as a software platform in VLs.Ogre3D is a 3D graphics rendering engine as the image rendering core. Auxiliary functions is completed by a variety of $\mathrm{C}++$ class library. The virtual environment model is built in Solidworks and rendered in 3DsMax, which is finally presented in the virtual environment. 
As the core of VLs, the mathematical model is calculated in the mathematical software Mathmacitca or Matlab.Virtual experiment is drived by the validated mathematical model.

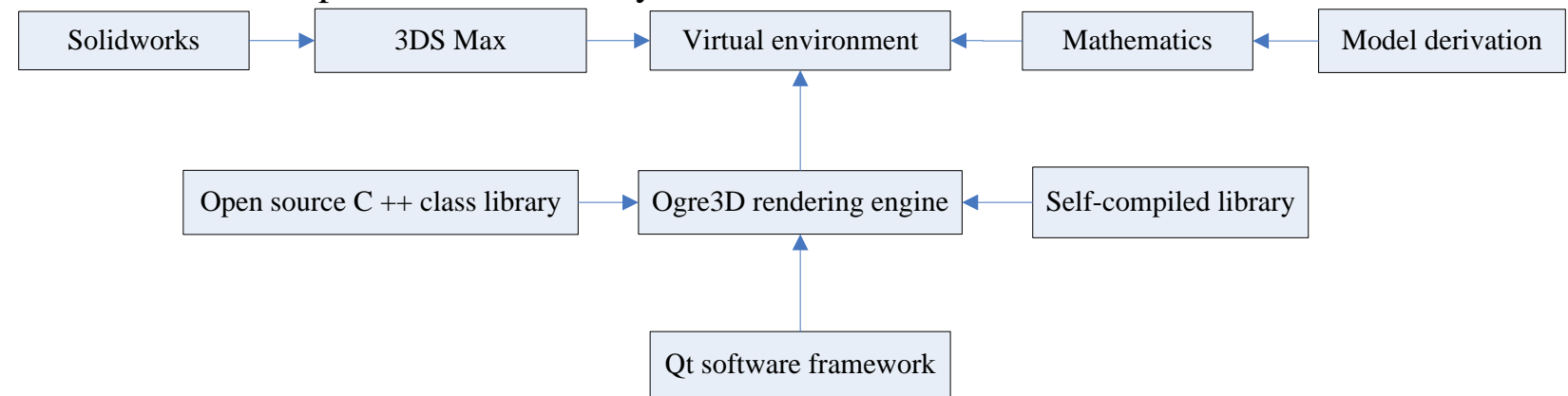

\subsection{Robot Kinematics}

Fig. 1 Architecture of Virtual Labs

The virtual laboratory focus on the Transformations, Homogeneous Transformation, DenavitHartenberg Convention, Forward Kinematics and Jacobian.

I: Transformations: it has the following components.

a. Translation: the 'Position' of any point, $\mathrm{P}$, on a rigid body in motion with respect to the fixed reference frame can be described by the 3-dimentional Cartesian vector-, it is denoted as:

$$
[\boldsymbol{p}]_{F}=\left[\begin{array}{l}
p_{x} \\
p_{y} \\
p_{z}
\end{array}\right]
$$

b. Rotation: the 'Orientation' of a rigid body with respect to the fixed frame can be described in different ways. Direction Cosine Representation and Euler Angle Representation.

Direction Cosine Representation: $\mathrm{Q}$ matrix is the rotation or orientation matrix transforming the representation of vector from frame $\mathrm{M}$ to $\mathrm{F}$, they are given as follows:

$$
[p]_{F}=Q[p]_{M}
$$

And

$$
\boldsymbol{Q}=\left[\begin{array}{lll}
u_{x} & v_{x} & w_{x} \\
u_{y} & v_{y} & w_{y} \\
u_{z} & v_{z} & w_{z}
\end{array}\right]
$$

Only three parameters are independent and should be sufficient to define the three-DOF rotational motion, and it is difficult to choose the set of three independent parameters. This is the drawback.

Euler Angle Representation: the ZYZ set is commonly used in the Euler angle representation, it means:

(1) Rotate the fixed frame F, by the angle ${ }^{\emptyset}$, about the axis Z:

$$
\boldsymbol{Q}_{z}=\left[\begin{array}{ccc}
C \theta & -S \theta & 0 \\
S \theta & C \theta & 0 \\
0 & 0 & 1
\end{array}\right]
$$

(2) Rotate the current frame by an angle ${ }^{\theta}$ about its $Y^{*}$ axis.

$$
\boldsymbol{Q}_{Y^{\prime}}=\left[\begin{array}{ccc}
C \theta & 0 & S \theta \\
0 & 1 & 0 \\
-S \theta & 0 & C \theta
\end{array}\right]
$$

(3) Rotate the current frame by an angle ${ }^{\theta}$ about its $Z^{z *}$ axis.

$$
Q_{Z^{s}}=\left[\begin{array}{ccc}
C \theta & -S \theta & 0 \\
S \theta & C \theta & 0 \\
0 & 0 & 1
\end{array}\right]
$$

The resulting orientation of the frame $\mathrm{M}$ as denoted by $\mathrm{Q}$, is obtained from the composition of the three elementary rotations $\boldsymbol{Q}_{z}, \boldsymbol{Q}_{Y^{\prime}}, \boldsymbol{Q}_{Z^{E}}$ :

$$
Q=Q_{z} Q_{Y^{\prime}} Q_{Z^{\prime \prime}}
$$


The drawback is that it sometimes fails in finding the solution of an inverse problem.

II: Homogeneous Transformation: ${ }^{4 \times 4}$ matrix $\mathrm{T}$ includes both translation and rotation from frame $\mathrm{M}$ to $\mathrm{F}$ is shown:

$$
T=\left[\begin{array}{ll}
Q & P \\
0 & 1
\end{array}\right]
$$

It is used to calculate the new coordinate values for a robot part.

III: Denavit-Hartenberg Convention: DH Convention is used for selecting frames of reference in robotics, and it relays on how to set the frame. It is convenient to set some rules for the definition of the link frames:

a. Let axis denotes the axis of the joint connecting link $i-1$ to link ${ }^{i}$;

b. A coordinate system is attached to the end of the link ${ }^{i-1}$;

c. Choose the axis $Z_{\tilde{i}}$ along the axis of joint;

d. Locate the origin ${ }^{O_{i}}$, at the intersection of axis ${ }^{Z_{i}}$ with the common normal to $Z_{\bar{i}-1}$ and $Z_{i \bar{i}}$

e. Choose the axis $X_{\tilde{i}}$ along the common normal to axes ${ }^{Z_{i-1}}$ and ${ }^{Z_{\tilde{i}}}$ with the direction from former to the later.

f. Choose axis $Y_{\tilde{i}}$ so as to complete a right-handed frame.

Thus, the four DH parameters are defined as follows:

(a) $b_{i}$ : This is measured as the distance between $X_{\tilde{i}}$ and $X_{i+1}$ along $Z_{\tilde{i}}$

(b) $\theta_{i}$ : This is measured as the angle between $X_{\bar{i}}$ and $X_{i+1}$ along $Z_{i}$

(c) $a_{\tilde{i}}$ : This is measured as the distance between the common normal to the axes $Z_{\tilde{i}}$ and $Z_{\tilde{i}+1}$ about $X_{i+1}$

(d) $\alpha_{i}$ : This is measured as the angle between the axes $Z_{i}$ and $Z_{i+1}$ about $X_{i+1}$

It is possible to express the coordinate transformation between the $\mathrm{DH}$ frame ${ }^{i}$ to frame ${ }^{i+1}$ :

$$
{ }_{i+1}^{i} \boldsymbol{T}=\left[\begin{array}{cccc}
C \theta_{\tilde{i}} & -S \theta_{i} C \alpha_{i} & S \theta_{i} S \alpha_{i} & a_{i} c \theta_{i} \\
S \theta_{i} & C \theta_{i} C \alpha_{i} & -C \theta_{i} S \alpha_{i} & a_{i} S \theta_{i} \\
0 & S \alpha_{i} & C \alpha_{i} & b_{i} \\
0 & 0 & 0 & 1
\end{array}\right]
$$

IV: Forward Kinematics: In the forward or direct kinematics for positions, the joint positions, i.e. the angles of the revolute joints and the displacements of the prismatic joints, are prescribed.

(a) Attach a coordinate frame to each of links;

(b) Define the DH parameters.

(c) Write the homogeneous transformation matrices: ${ }_{2}^{1} T,{ }_{a}^{2} T \ldots{ }^{n+1} T$

(d) ${ }^{n+1} T$ is now obtained by the post-multiplication of the above individual homogeneous transformations:

$$
{ }_{n+1}^{1} T={ }_{2}^{1} T{ }_{3}^{2} T \cdots{ }_{n+1}^{n} T
$$

V: Jacobian:

The Jacobian constitutes one of the most important tools for manipulator characterization. Jacobian matrix $l$, can be extracted as

$$
\mathrm{J}=\left[\begin{array}{cccc}
e_{1} \times a_{1 e} & e_{2} \times a_{2 e} & \cdots & e_{n} \\
e_{1} & e_{2} & \cdots & e_{n} \times a_{n e}
\end{array}\right]
$$

Where

$e_{i}$ is the unit vector parallel to the translational direction of link ${ }^{i}$ with respect to $\operatorname{link}^{i-1}$;

$\boldsymbol{a}_{i j, j}$ is the vector joining the origin of link ${ }^{i}$ to the origin of link ${ }^{i-1}$;

Jacobian matrix $I$ is a function of the joint variables. 


\subsection{Experiment Planning and Execution}

The virtual lab is created to study the above mentioned concept for six DOF manipulator. Steps to do the experiment are as follows:

Step-1: coordinate systems are attached to every link of the manipulator;
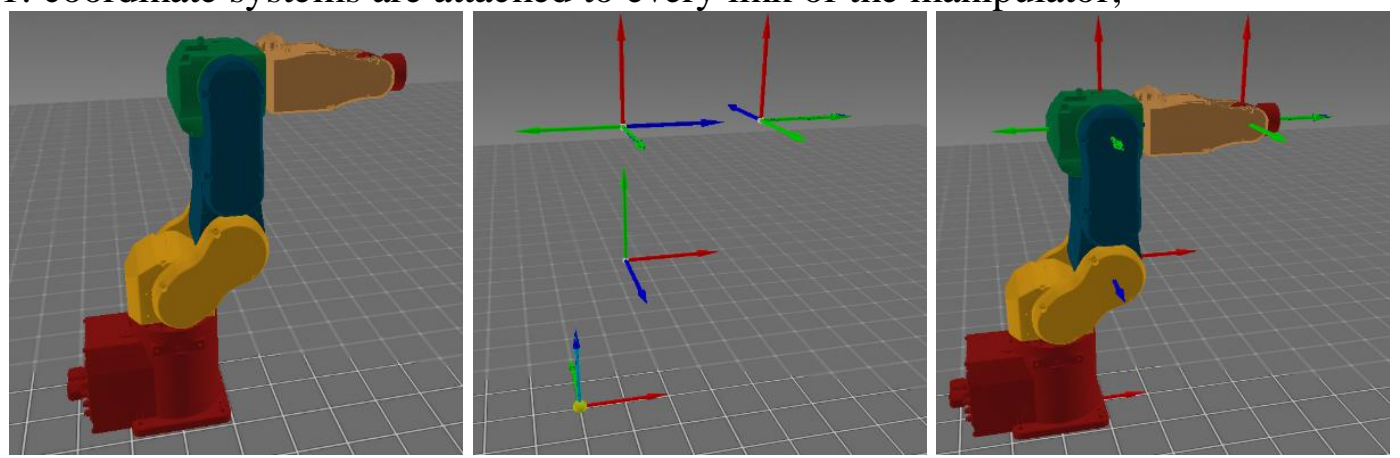

Fig.2 6 DOF manipulator and attached coordinates

Step-2: Calculate and display the DH parameters

\begin{tabular}{|l|l|l|l|l|}
\hline \multicolumn{5}{|c|}{ DH parameters } \\
\hline $\mathrm{i}$ & \multicolumn{2}{|c|}{ bi } & \multicolumn{1}{|c|}{$\theta \mathrm{i}$} & \multicolumn{2}{c|}{ ai } & \multicolumn{1}{c|}{ ai } \\
\hline 1 & 315 & 0 & 100 & -90 \\
\hline 2 & 0 & 90 & 250 & 0 \\
\hline 3 & 0 & 0 & 0 & 90 \\
\hline 4 & 250 & 0 & 0 & -90 \\
\hline 5 & 0 & 0 & 0 & 90 \\
\hline 6 & 0 & 0 & 0 & 0 \\
\hline
\end{tabular}

Fig. 3 The DH parameters

Step-3: Calculate and display the forward kinematics of robot;

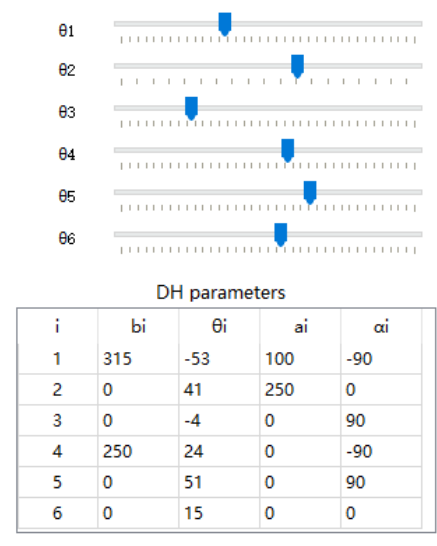

\begin{tabular}{|c|c|c|c|}
\multicolumn{4}{c|}{ Homogeneous Transformation } \\
\hline X & $Y$ & Z & \multicolumn{1}{c|}{$P$} \\
-0.4419 & -0.8393 & 0.3167 & 264.2759 \\
\hline-0.2174 & -0.2424 & -0.9455 & -350.7056 \\
\hline 0.8704 & -0.4866 & -0.0753 & 279.3559 \\
\hline 0.0000 & 0.0000 & 0.0000 & 1.0000 \\
\hline
\end{tabular}

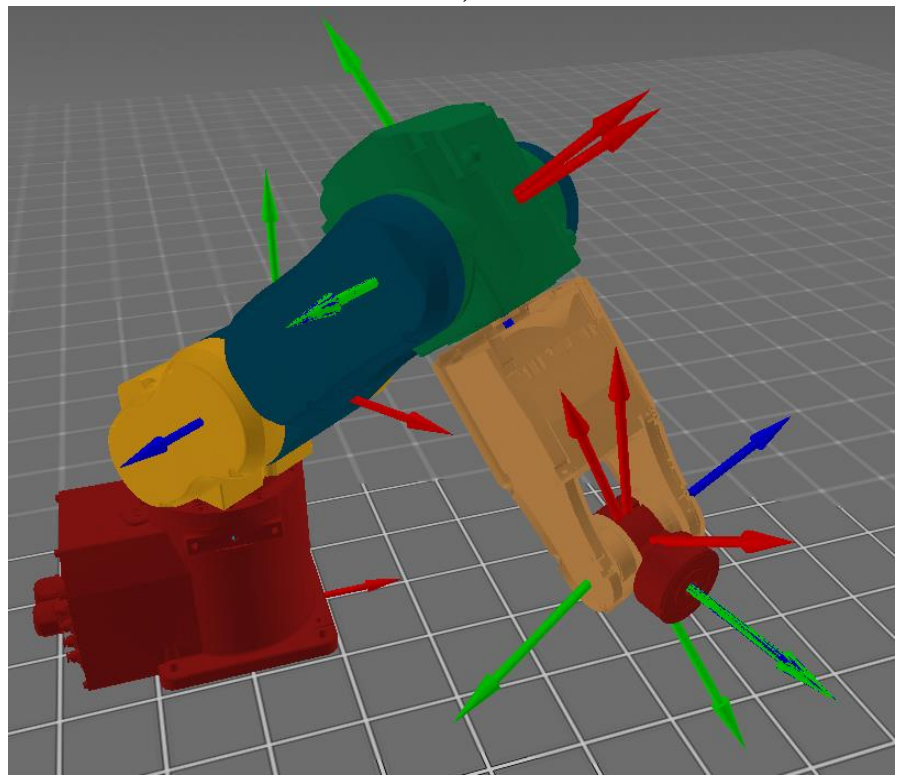

Fig.4 The kinematics of robot manipulato

Step-4: Calculate and display the Jacobian matrix; 


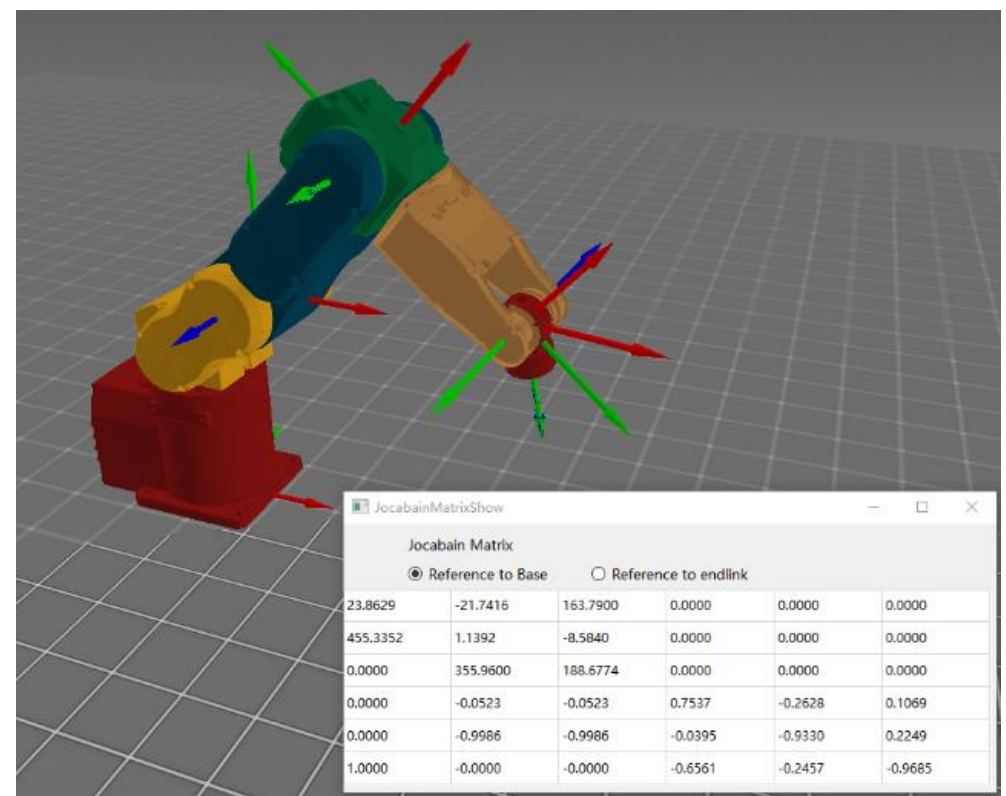

Fig. 5 Jacobian matrix of robot manipulator

Step-5: Control the joint variables and observe the change of DH parameters, forward Kinematics, and Jacobian.

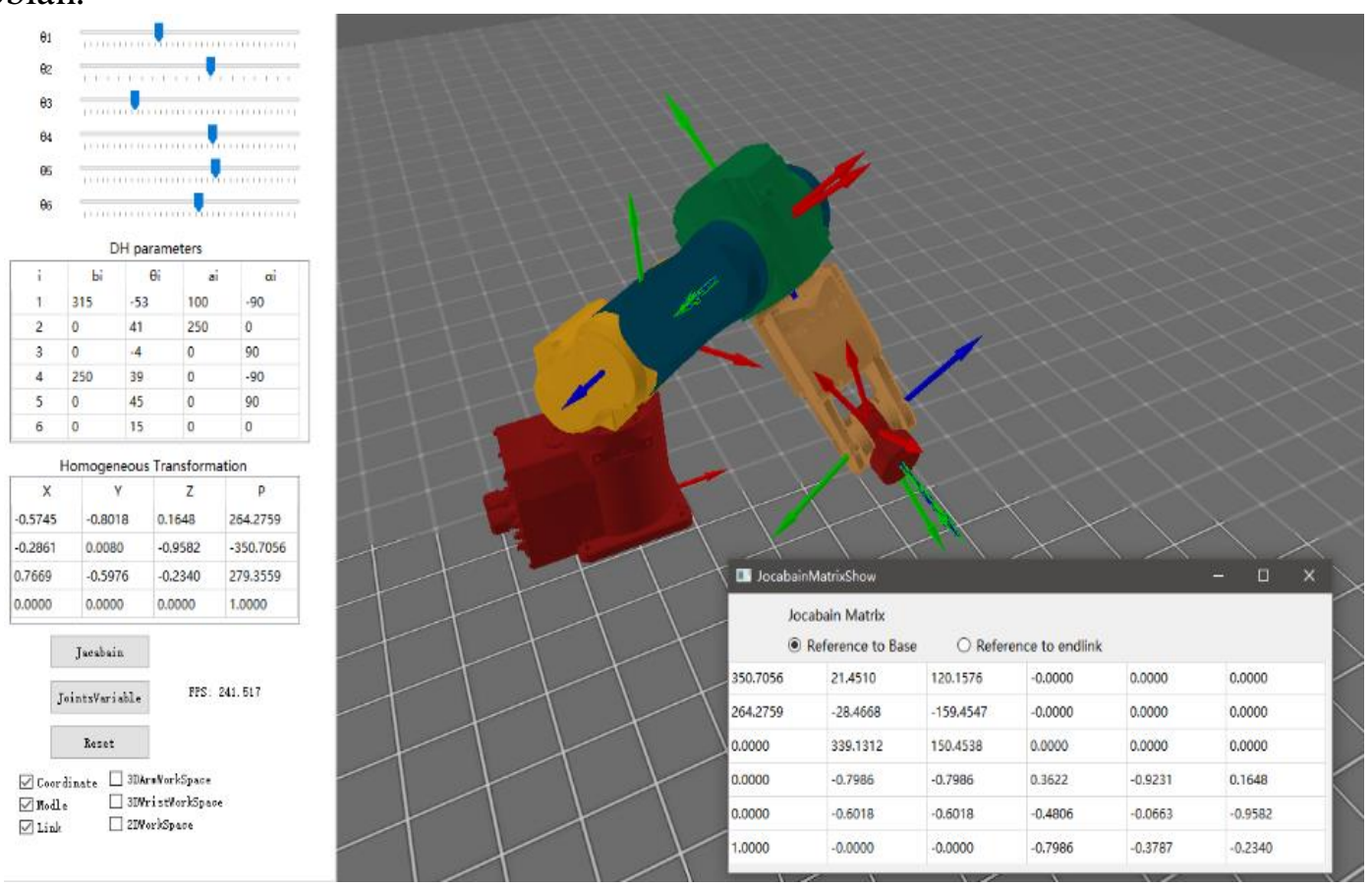

Fig. 6 Changing the input and observing the motion

\section{Conclusion}

The virtual laboratory of coordinate transformation has the characteristics of user-friendly interface, clear process simulation and accurate operation result. The software system based on the Qt software framework and the Ogre3D rendering engine communicates well with user and hardware. The theoretical knowledge involved in the real experiment is presented through the virtual experiment, which can speed up the students 'learning progress and deepen the students' understanding, and lay a good foundation for the study of robotics. 


\section{References}

[1]. Carlos A Jara, Francisco A Candelas, Pablo Gil, Fernando Torres, Francisco Esquembre, Sebasti án Dormido. EJS+EjsRL: An interactive tool for industrial robots simulation, Computer Vision and remote operation [J]. Robotics \& Autonomous Systems, 2011, 59(6): 389-401.

[2]. A Gil, A Peidró, Ó Reinoso, J M Marín. Implementation and assessment of a virtual laboratory of parallel robots developed for engineering students [J]. IEEE Transactions on Education, 2014, 57(2): 92-98.

[3]. H M, M H F, Bal M. A Modular Virtual Reality System for Engineering Laboratory Education [J].Computer Applications in Engineering Education, 2011, 19(2): 305-314.

[4]. Mark, W, Spong, Seth, HUtchinson, M, Vidyasagar. Robot Modeling and control [M]. NewYork:John Wiley \& Sons, 2006. 